\title{
THREATENED LICHENS OF LOWER SILESIA, POLAND
}

\author{
MARIA KOSSOWSKA ${ }^{1}$, JERZY FABISZEWSKI ${ }^{2}$ \\ ${ }^{1}$ Department of Systematics and Phytosocjology \\ Institute of Plant Biology, Wrocław University \\ Kanonia 6/8, 50-328 Wrocław, Poland \\ e-mail: kossmar@biol.uni.wroc.pl \\ ${ }^{2}$ Department of Botany and Plant Physiology, Agricultural University \\ Cybulskiego 32, 50-205 Wrocław, Poland
}

(Received: July 4, 2003. Accepted: February 2, 2004)

\begin{abstract}
A preliminary red list of threatened lichens of Lower Silesia (SW Poland) is presented. Species have been valuated according to the Red Data Book Categories (IUCN). The list comprises 602 taxa (ca. $60 \%$ of the whole lichen flora of Lower Silesia). 52 species are considered as rare (category R), 84 - as vulnerable (V), and 63 species - as endangered (E). The Silesian lichen flora is characterized by great numbers of taxa with extinct (Ex 140 taxa), and indeterminate (I - 263 taxa) categories.
\end{abstract}

KEY WORDS: threatened lichens, red list, Lower Silesia, Poland.

\section{INTRODUCTION}

Two subsequent editions of Polish red list of threatened lichens published recently (Cieśliński et al. 1986, 1992) drew lichenologists' attention of to a process of the extinction of lichen species and inspired them to prepare similar lists for micro- and macroregions, mountain ranges etc. (e.g., Leśniański 1998; Śliwa 1998; Kiszka and Leśniański 1999). These lists will supplement the synthetic Polish list with detailed descriptions, showing local differences in both a kind and degree of threat factors.

In 1997, at the annual meeting, Polish lichenologists passed a resolution to make red lists for all regions of Poland distinguished as physical-geographic subprovinces according to Kondracki $(1988,1998)$. All these lists should have been published together in a one book edition. This plan has not been executed so far, but some results have been already achieved, among others presented regional red list of threatened lichens of Lower Silesia. It should be considered as preliminary and rough one. In the future, when our knowledge about contemporary conditions of lichen flora increases, the list will be verified.

The presented list bases on a preliminary check-list of Lower Silesian lichens (Kossowska, in preparation), containing all species reported from this area and mentioned in literature. Untill now the preliminary check-list is composed of ca. 1020 species, i.e. more than $60 \%$ of the whole Polish lichen flora (see Fałtynowicz 1993). Such a great number of recorded lichen taxa is caused by the abound- ance and diversity of potential habitats, as well as by careful investigations carried out in the past. The lichen flora of Lower Silesia is one of the better recognized in Poland. A considerable part of records dates from the 19th and the beginning of the 20th centuries and was shed by eminent German lichenologists, e.g. Flotow 1849, 1850; Koerber 1855; Stein 1879, 1889; Eitner 1896, 1901, 1911, and others. Thanks to them and subsequent works of Polish lichenologists (Tobolewski 1855; Fabiszewski 1962, 1968) we have obtained nearly complete knowledge about Lower Silesian lichen flora before the great anthropogenic extinction, started in the seventies of the 20th century.

Names of lichen species are quoted according to Santesson (1993) and Fałtynowicz (1993).

\section{LOCATION AND BORDERS OF THE STUDIED AREA}

Lower Silesia (Dolny Śląsk, Niedere Schlesien) is a historical and geographical province in the south-western part of Poland, bordered upon Germany and Czech Republic. Due to the recent administrative division of the country one big voivodship with the capital city - Wrocław was established in this region; the borders of this voivodship were accepted as conventional borders of the area taken into consideration in this work (Fig. 1).

Such a delimited study area involves following physicalgeographic subprovinces (according to Kondracki 1998): 


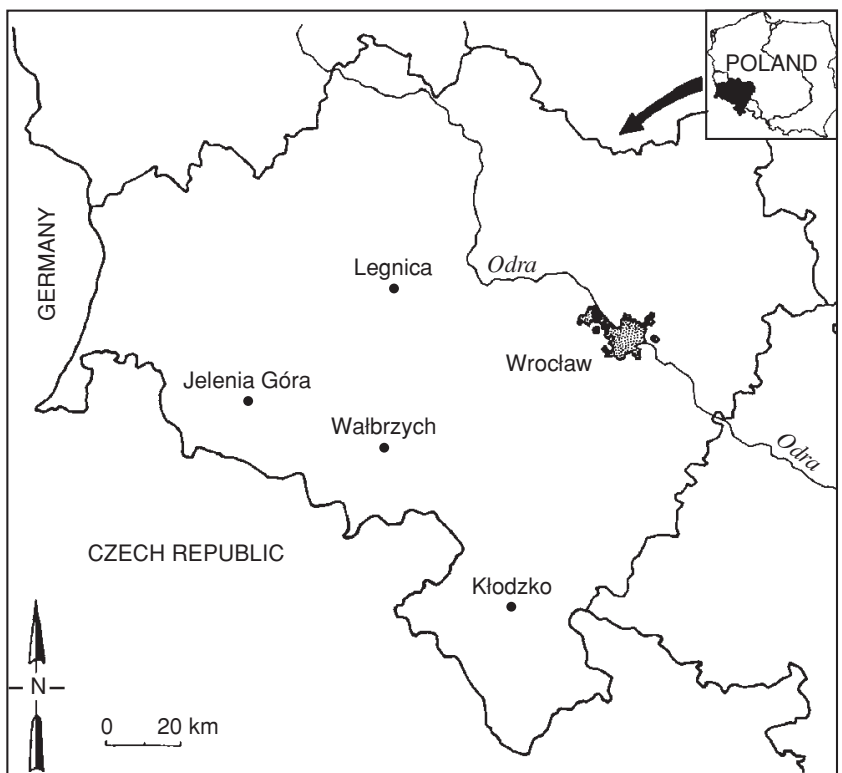

Fig. 1. Location and borders of province of Lower Silesia.

the Sudety Mts., the Śląsko-Łużyckie Lowlands and a part of the Srodkowopolskie Lowlands.

\section{CATEGORIES OF THREAT}

Categories used for a valuation of a species threat degree have to be briefly explained. In December 1994, the World Conservation Union (IUCN) replaced hitherto used the Red Data Book Categories by a new system of threat categories and recommended them for use in new versions of red lists and red books. This system is composed of eight categories and three subcategories of threat, and of precise criteria of a valuation. Those new categories have been named as the Red List Categories (see IUCN Red List Categories...).

The clearly defined valuation criteria were assumed to make preparation of red lists easier and more objective. However, their strict application to lichens seems to be very difficult or even impossible, mainly because of lack of precise information about a current and historical size of populations, a number of mature individuals etc. As a result, according to the recommended criteria most of the lichen species should be considered as data deficient (category DD).

Therefore, in the presented list the authors decided to use the previous Red Data Book Categories (Cieśliński et al. 1992), as follows:

- Ex (Extinct) - Species which are not confirmed after repeated searches either on known localities or in similar habitats.

- E (Endangered) - Taxa in danger of extinction and which survival is hardly probable if the casual factors continue operating.

- V (Vulnerable) - Species belived to move to the endangered category in near future if the casual factors continue operating

- R (Rare) - Species of limited geographical range, small habitat areas or occuring on wide areas, but in considerable dispersion. At present, they are neither endangered nor vulnerable, but are at risk.
- I (Indeterminate) - Taxa known to be extinct, endangered, vulnerable or rare, but there are insufficient data to say which category is appropriate.

Advantage of such a solution is possibility of comparing our list with existing regional lists and the Polish list. However, lack of compatibility with new instructions of IUCN is its unquestionable drawback. Therefore, in the future, lichelologists who will study the lichen flora of Lower Silesia ought to pay particular attention to size and dynamics of species populations, especially of rare and endangered taxa. Consequently, in the second edition of our red list, we will be able to valuate lichen taxa according to the Red List Categories.

\section{SHORT DISCUSSION}

The red list of threatened lichens of Lower Silesia comparises 602 taxa, that is ca. $60 \%$ of the total lichen flora of this region. Among them, 52 species are considered as rare (category R), 83 - as vulnerable (V) and 63 species - as endangered $(\mathrm{E})$. The characteristic trait of the Lower Silesian lichen flora are particularly numerous extinctas well as indeterminate species (140 and 264 respectively). Taxa with these two categories compose together two thirds of all species included to the red list.

A group of extinct taxa is mainly constituted of lichens especially sensitive to air pollution, e.g. macrolichens from the genera Alectoria, Bryoria, Evernia, Lobaria, Nephroma, Ramalina, Sticta and Usnea. These species are particularly endangered everywhere within their geographical ranges; some of them have no present locality in Poland (category Ex in the Polish red list - Cieśliński et al. 1992). The other group of taxa considered as extinct is composed of lichens known only from single localitites and occupying very specific habitats, e.g., aquatic species or lichens - relicts of a primeval forest (acc. to Cieśliński et al. 1996). The remaining species, that have not been recorded for a long time were considered as „lost” but still possible to be recovered and have got a category I. A great number of indeterminate species is resulted from, mentioned above, careful lichenological investigations carried out in Lower Silesia in the past. Many inconspiciuous species, easy to overlook were then recorded in single sites. Some of them could die out, but it is possible that the other still persist at the same or similar localities. Thus, the category I should be considered as a temporary one. In the future, the species included here shall be either moved to another category, or, what seems to be less probable, excluded from the list as not endangered.

When one compare the presented list with the Polish one, the lichen flora of Lower Silesia seems to be much more endangered (Fig. 2). It is probably caused by synanthropization of the environment, which has started earlier and has been more intensive than in other parts of Poland. Anthropogenic transformations in this region include mainly urbanization and industrialization processes, resulted in air and water pollution, forest felling, exploitation of natural rock outcrops and many others. In the seventies of the $20^{\text {th }}$ century the local air pollution caused by emission of gases such as $\mathrm{SO}_{2}, \mathrm{NH}_{\mathrm{x}}$ and $\mathrm{HF}$ and dust contamination were intensified by a large-scale air pollution come from above the territories of former Czechoslovakia and German 


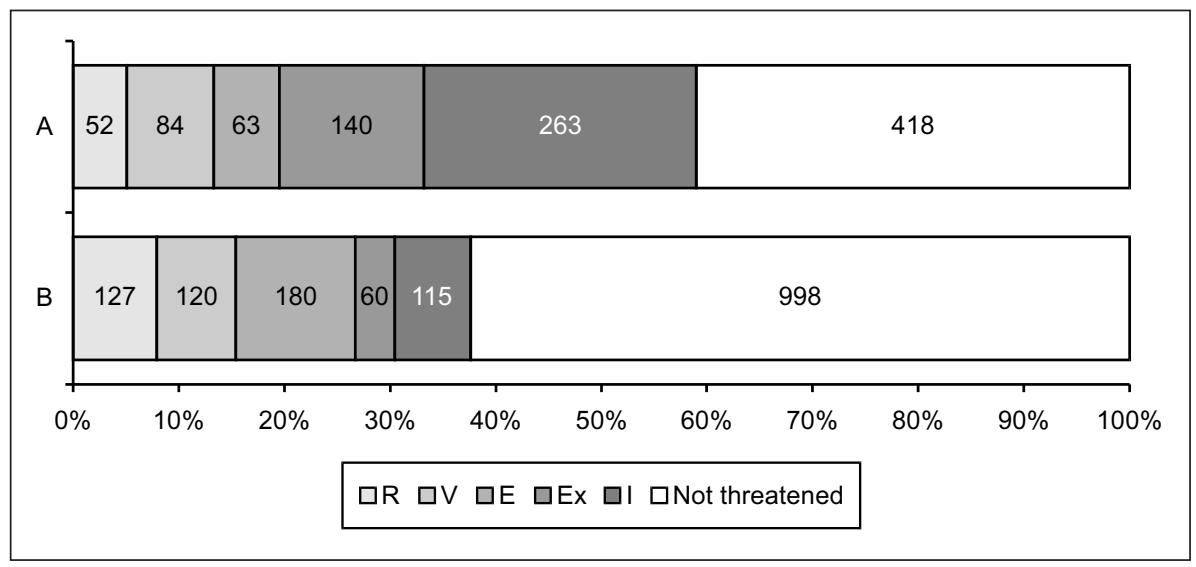

Fig. 2. Number of species and percentage of each category of threat in lichen flora of Lower Silesia (A) and Poland (B).
Democratic Republic. It was the main reason of the ecological disaster of mountain forests in the Sudetes.

All those factors exerted a strong harmful impact on the Lower Silesian lichens, resulted in a considerable decline of a number of species. A relatively reach lichen flora could persist only in small refuges, characterised by a peculiar combination of habitat factors and usually protected from air pollution by specific topographic features.

\section{LITERATURE CITED}

CIEŚLIŃSKI S., CZYŻEWSKA K., FABISZEWSKI J. 1986. Czerwona lista porostów zagrożonych w Polsce. In: Zarzycki K., Wojewoda W. (eds), Lista roślin wymierających i zagrożonych w Polsce. Wyd. 1. PWN, Warszawa, p. 83-107.

CIEŚLIŃSKI S., CZYŻEWSKA K., FABISZEWSKI J. 1992. Czerwona lista porostów zagrożonych w Polsce. In: Zarzycki K., Wojewoda W., Heinrich Z. (eds), Lista roślin zagrożonych w Polsce. Ed. 2. Instytut Botaniki PAN, Kraków, p. 57-74.

CIEŚLIŃSKI S., CZYŻEWSKA K., FALIŃSKI J. B., KLAMA H., MUŁENKO W., ŻARNOWIEC J. 1996. Relikty lasu puszczańskiego. Zjawiska reliktowe (wyniki badań w Projekcie CRYPTO na stałej powierzchni BSG: V-100; BPN; oddz. 256). Phytocenosis 8 (N.S.), Semin. Geobot. 4: 47-64.

EITNER E. 1896. Nachträge zur Flechtenflora Schlesiens. Jahresb. Schles. Ges. vaterl. Kultur 73: 2-26.

EITNER E. 1901. II Nachtrag zur Schlesischen Flechtenflora. Jahresb. Schles. Ges. vaterl. Kultur 78: 5-27.

EITNER E. 1911. Dritten Nachtrag zur Schlesischen Flechtenflora. Jahresb. Schles. Ges. vaterl. Kultur 88(1): 20-60.

FABISZEWSKI J. 1962. Flora porostów nadrzewnych masywu Ślęży. Fragm. Flor. Geobot. 8(1): 57-66. (in Polish with English summary)

FABISZEWSKI J. 1968. Porosty Śnieżnika Kłodzkiego i Gór Bialskich. Monogr. Bot. 26: 1-115. (in Polish with French summary)
FAŁTYNOWICZ W. 1993. A checklist of polish lichen forming and lichenicolous fungi including parasitic and saprophytic fungi occurring on lichens. Polish Bot. Stud. 6: 1-65.

FLOTOW J. VON. 1849. Lichenes Florae Silesiae. I. Jahresb. Schles. Ges. vaterl. Kultur 27: 98-135.

FLOTOW J. VON. 1850. Lichenes Florae Silesiae. II. Jahresb. Schles. Ges. vaterl. Kultur 28: 115-143.

IUCN List Categories. Prepared by the IUCN Species Survival Commission. As approved by the $40^{\text {th }}$ Meeting of the IUCN Council Gland, Switzerland, 30 Nov. 1994.

KISZKA J., LEŚNIAŃSKI G. 1999. Czerwona lista porostów Górnego Śląska. Centrum Dziedzictwa Przyrody Górnego Śląska. Raporty i Opinie 4: 52-110.

KOERBER G.W. 1855. Systema Lichenum Germaniae. Die Flechten Deutschlands, insbesondere Schlesiens. E. Trewendt \& Granier Verl., Breslau.

KONDRACKI J. 1988. Geografia fizyczna Polski. PWN, Warszawa. (in Polish)

KONDRACKI J. 1998. Geografia regionalna Polski. PWN, Warszawa. (in Polish)

LEŚNIAŃSKI G. 1998. Lokalna czerwona lista porostów zagrożonych na Śląsku Opolskim. In: Czyżewska K. (ed.), Różnorodność biologiczna porostów. Wyd. Uniwersytetu Łódzkiego, Łódź, p. 57-63.

SANTESSON R. 1993. The lichens and lichenicolous fungi of Sweden and Norway. SBT, Lund.

STEIN B. 1879. Flechten. In: Cohn's Kryptogamenflora von Schlesien. Jahresb. Schles. Ges. vaterl. Kultur 2(2): 1-400.

STEIN B. 1888. Nachträge zur Flechtenflora Schlesiens. Jahresb. Schles. Ges. vaterl. Kultur 66: 142-149.

ŚLIWA L. 1998. Czerwona lista porostów zagrożonych Beskidu Sądeckiego. In: K. Czyżewska (ed.), Różnorodność biologiczna porostów. Wyd. Uniwersytetu Łódzkiego, Łódź, p. 57-74.

TOBOLEWSKI Z. 1955. Porosty Gór Stołowych. Prace Kom. Biol. PTPN 16 (1): 1-99. 


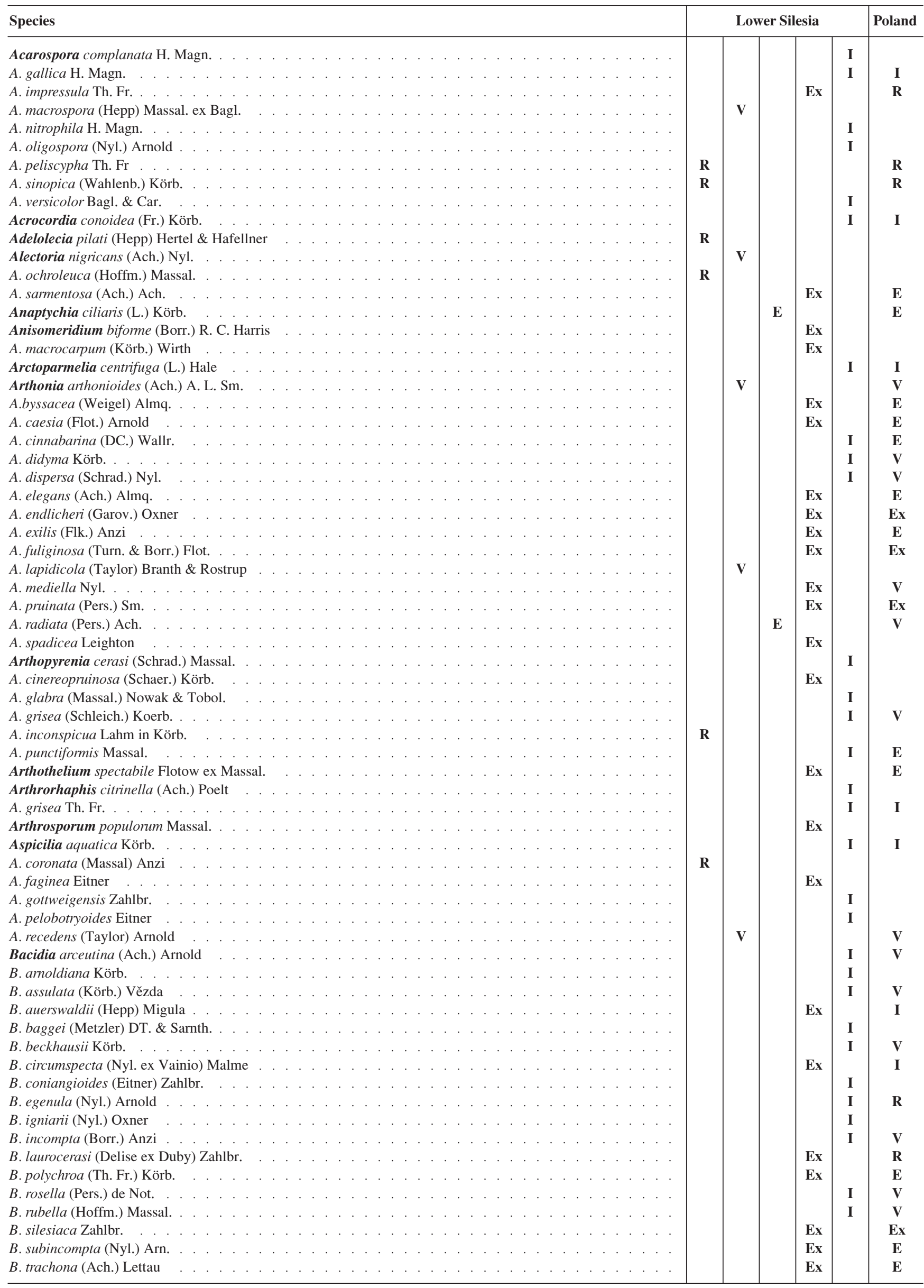




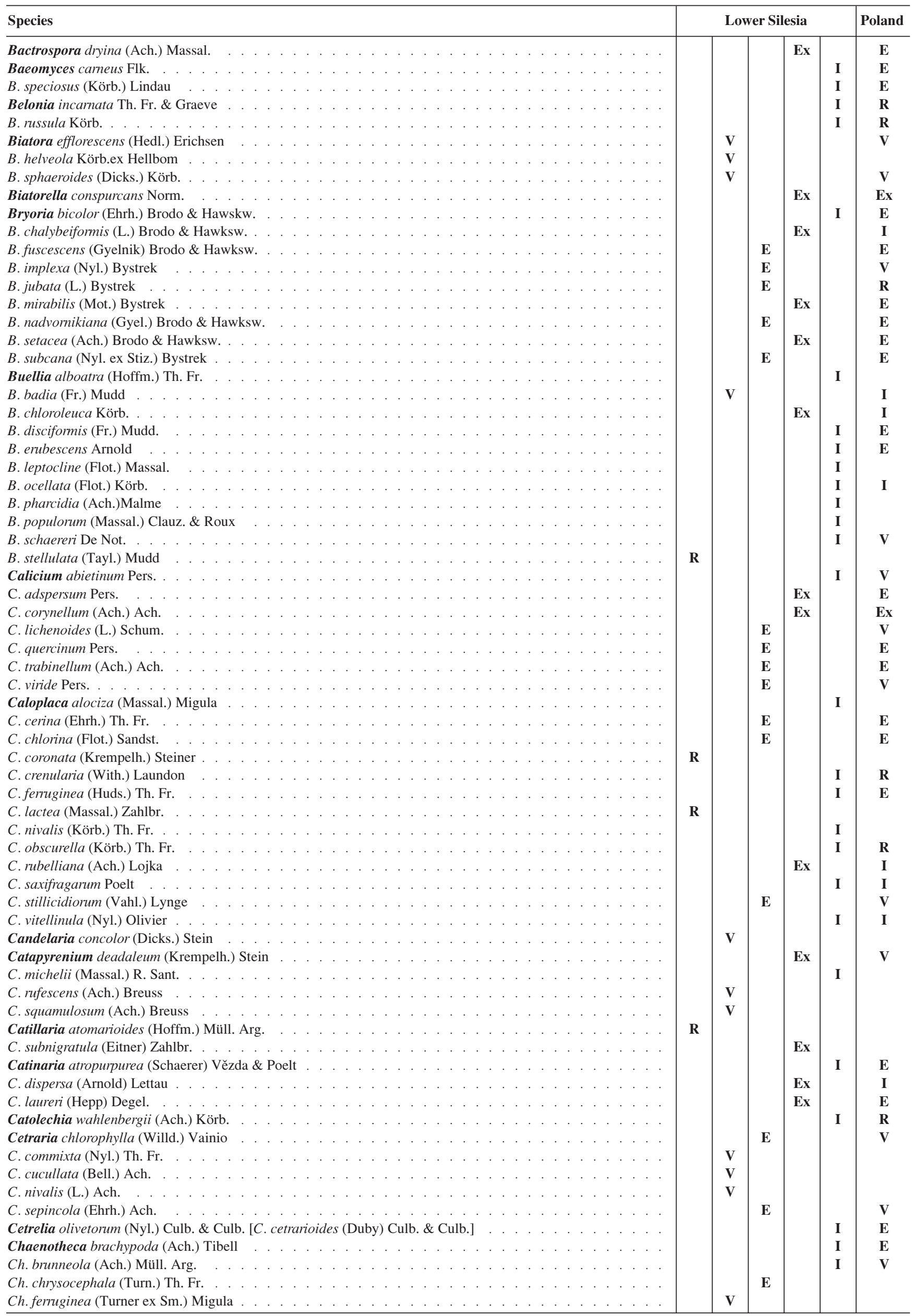


Ch. furfuracea (L.) Tibell

Ch. phaeocephala (Turn.) Th. Fr.

Ch. stemonea (ach.) Müll. Arg.

Ch. trichialis (Ach.) Th. Fr.

Ch. xyloxena Nadv.

Chrysotrix candelaris (L.) Laundon

Cladonia botrytes (Hagen) Willd.

C. brevis Sandst.

C. cariosa (Ach.) Spreng.

C. carneola (Fr.) Fr.

C. cyanipes (Sommerf.) Nyl.

C. decorticata (Flk.) Spreng.

C. macroceras (Delise) Hav.

C. macrophylla (Schaer.) Stenham.

C. parasitica (Hoffm.) Hoffm.

C. ramulosa (With.) Laundon

C. sulphurina (Michx.) Fr.

C. turgida Hoffm.

Clauzadeana macula (Taylor) Coppins \& Rambold [Aspicilia morioides Blomb.]

Cliostomum corrugatum (Ach.) Fr.

C. griffithii (Sm.) Coppins

Collema auriforme (With.) Coppins \& Laundon

C. callopismum Massal.

C. coccophorum Tuck.

C. conglomeratum Hoffm.

C. crispum (Huds.) Weber

C. cristatum (L.) Weber

C. dichotomum (With.) Coppins \& Laundon

C. flaccidum (Ach.) Ach.

C. fragrans (Sm.) Ach.

C. glebulentum (Nyl. ex Crombie) Degel.

C. limosum (Ach.) Ach.

C. nigrescens (Huds.) DC.

C. occultatum Bagl.

C. polycarpon Hoffm.

Cornicularia normoerica (Gunn.) Du Rietz

Cresporhaphis muellerii (Duby) Aguirre

C. weinkampii (Lahm) Aguirre

Cyphelium notarisii (Tul.) Blomb. \& Forssell

C. tigillare (Ach.) Ach.

Dermatocarpon luridum (With.) Laundon

Dimelaena oreina (Ach.) Norm.

Dimerella lutea (Dicks.) Trevisian

Diploicia canescens (Dicks.) Massal.

Diploschistes euganeus (Massal.) Stnr.

D. gypsaceus (Ach.) Zahlbr.

Endocarpon adscendens (Anzi) Müll. Arg.

E. pusillum Hedw.

Enterographa hutchinsiae (Leight.) Massal.

Eopyrenula leucoplaca (Wallr.) R. C. Harris

Ephebe lanata (L.) Vainio

Evernia divaricata (L.) Ach.

E mesomorpha $\mathrm{Nyl}$.

E. prunastri (L.) Ach.

Farnoldia hypocrita (Massal.) Fröberg

$F$. jurana (Schaer.) Hertel

Flavoparmelia caperata (L.) Hale

Fritzea lamprophora (Körb.) Stein

Fulgensia fulgens (Swartz) Elenkin

Fuscidea austera (Nyl.) P. James

F. kochiana (Hepp) Wirth \& Vězda

F. lightfootii (Sm.) Koppins \& James

$F$. recensa (Stirton) Hertel, Wirth \& Vězda

Graphis scripta (L.) Ach.

Gyalecta truncigena (Ach.) Hepp

G. ulmi (Sw.) Zahlbr.

Gyalidea diaphana (Körb.) Vězda

G. fritzei (Stein) Vězda

Haematomma ochroleucum (Neck.) Laund.

Harpidium rutilans (Flot.) Körb.

I


Helocarpon crassipes Th. Fr.

Heppia lutosa (Ach.) Nyl.

Hymenelia lacustris (With.) Choisy

Hyperphyscia adglutinata (Flk.) Mayrh. \& Poelt

Hypogymnia farinacea Zopf

H. tubulosa (Schaer.) Hav.

H. vittata (Ach.) Parr.

Hypotrachyna revoluta (Flk.) Hale

H. sinuosa (Sm.) Hale

Icmadophila ericetorum (L.) Zahlbr.

Imshaugia aleurites (Ach.) S. L. F. Meyer

Ionaspis chrysophana (Körb.) Stein

I. epulotica (Ach.) Arnold

I. odora (Ach.) Stein

Körberiella wimmeriana (Körb.) Stein

Lecanactis abietina (Ach.) Körb.

L. dilleniana (Ach.) Körb.

L. plocina Massal.

Lecania dubitans (Nyl.) A. L. Sm.

L. fuscella (Schaer.) Körb.

L. nylanderiana Massal.

L. quercicola Eitner

Lecanora achariana A. L. Sm.

L. albella (Pers.) Ach.

L. allophana (Ach.) Nyl.

L. argopholis (Ach.) Ach.

L. cateilea (Ach.) Massal.

L. demissa (Flot.) Zahlbr.

L. eitneriana Zahlbr.

L. epibryon (Ach.) Ach.

L. glabrata (Ach.) Malme

L. gypsodes Körb.

L. intumescens (Rebent.) Rabenh.

L. persimilis (Th. Fr.) Nyl.

L. subcarnea (Liljeblad) Ach.

L. subrugosa Nyl.

L. sulphurea (Hoffm.) Ach.

L. tephraea Körb. ex Stein

L. torquata (Fr.) Nyl.

L. umbrosa Degel.

Lecidea alboflava (Körb.) Arnold

L. atomaria $\mathrm{Th}$. Fr.

L. caesioatra Schaer.

L. decussata (Ach.) Th. Fr.

L. ecrustacea (Anzi ex Arnold) Arnold

L. exilis (Körb.) Rabenh.

L. fallax (Hepp) Linds.

L. limosa Ach.

L. lurida (Ach.) DC.

L. luteoatra Nyl.

L. nodulosa (Körb.) Olivier

L. ochrocarpa (Körb.) Lettau

L. phaeops $\mathrm{Nyl}$.

L. planorbis (Körb.) Lett.

L. pontifica (Körb. ex Stein) Zahlbr.

L. pycnocarpa (Körb.) Ohlert

L. ramulosa $\mathrm{Th}$. Fr.

L. silacea Ach.

L. squalescens Nyl.

L. steinii Zahlbr.

L. turgidula Fr.

Lecidella bullata Körb.

L. scabra (Taylor) Hertel \& Leuckert

Lemmopsis arnoldiana (Hepp) Zahlbr.

L. pelodes (Körb. ex Stein) L. T. Ellis

Lempholemma botryosum (Massal.) Zahlbr.

L. chalazanum (Ach.) B. de Lesd. 
Lower Silesia

L. corniculatum (Hoffm.) Minks.

L. cyanescens (Rabenh.) Körb.

L. intermedium (Arnold) Arnold

L. saturninum (Dicks.) Nyl.

L. schraderi (Bernh.) Nyl.

L. subtile (Swchrad.) Torss.

L. tenuissimum (Dickson) Körb.

Leptorhaphis quercus (Beltr.) Körb.

Letharia vulpina (L.) Hue

Lobaria linita (Ach.) Rabenh.

L. pulmonaria (L.) Hoffm.

L. scorbiculata (Scop.) DC.

Lobothalia radiosa (Hoffm.) Hafellner

Lopadium disciforme (Flot.) Kullhem

L. pezizoideum (Ach.) Körb.

Loxospora elatina (Ach.) Massal.

Maronea constans (Nyl.) Hepp

Massalongia carnosa (Dicks.) Körb

Megalaria grossa (Pers. ex Nyl.) Hafellner

M. pulverea (Borr.) Hafellner \& E. Schreiner

Megaspora verrucosa (Ach.) Hafellner \& Wirth

Melanelia elegantula (Zahlbr.) Essl.

M. exasperata (de Not.) Essl.

M. glabra (Schaer.) Essl.

M. panniformis (Nyl.) Essl.

M. sorediata (Ach.) Goward \& Ahti

M. stygia (L.) Essl.

M. subargentifera (Nyl.) Essl.

M. subaurifera (Nyl.) Essl.

Menegazzia terebrata (Hoffm.) Massal.

Micarea adnata Coppins

M. incrassata Hedl.

M. lignaria (Ach.) Hedl.

M. meleana (Nyl.) Hedl.

M. meleanida (Nyl.) Coppins

M. nitschkeana (Lahm. ex Rabenh.) Harm.

M. peliocarpa (Anzi) Coppins \& R. Sant.

M. turfosa (Massal.) Du Rietz

Miriquidica complanata (Körb.) Hertel \& Rambold

$M$. liljenstroemi Du Rietz

M. lulensis (Hellb.) Hertel \& Rambold

Moelleropsis nebulosa (Hoffm.) Gyeln.

Mycobilimbia lobulata (Sommerf.) Hafellner

M. microcarpa (Th. Fr.) Wirth

Mycoblastus sanguinarius (L.) Norm.

Nephroma bellum (Spreng.) Tuck.

$N$. laevigatum Ach.

N. parile (Ach.) Ach.

N. resupinatum (L.) Ach.

Normandina pulchella (Borrer) Nyl.

Ochrolechia pallescens (L.) Massal.

$O$. parella (L.) Massal.

O. subviridis (Hoeg.) Erichs.

O. tartarea (L.) Massal.

O. turneri (Sm.) Hasselrot

Opegrapha atra Pers.

O. calcarea $\mathrm{Sm}$.

O. dolomitica (Ach.) Koerb.

O. illecebrosa Dufour [L. amylacea (Ehrh.) Arnold]

O. ochrocheila Nyl.

O. rufescens Pers.

O. varia Pers.

$O$. vermicillifera (Kunze) Laundon

$O$. viridis (Pers.) Nyl.

O. vulgata Ach. var. vulgata [O. lithryga Ach.]

O. vulgata Ach. var. subsiderella Nyl. [O. niveoatra (Borr.) Laundon]

Pachyphiale carneola (Ach.) Arnold

Pannaria conoplea (Ach.) Bory

P. leucophaea (Vahl.) Jorg.

$P$. pezizoides (Web.) Trevisian

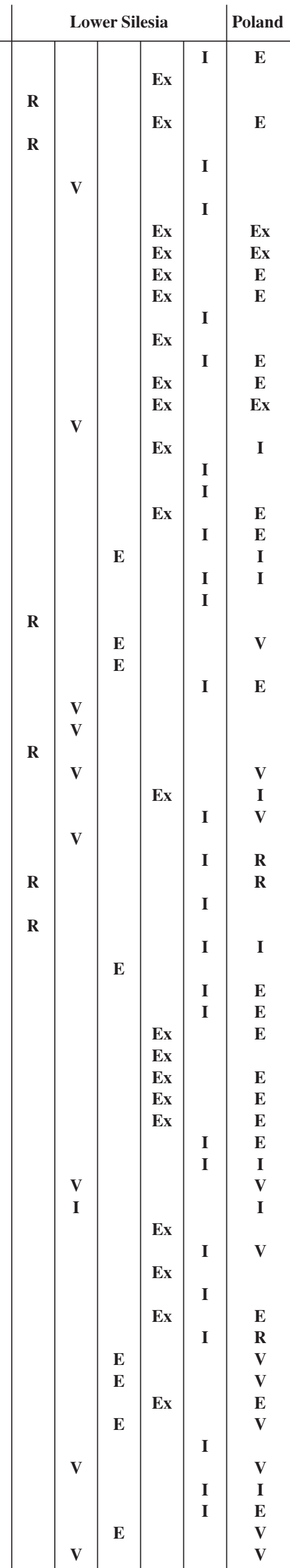


P. praetermissa Nyl. in Chyd. \& Furuhj.

Parmelia omphalodes (L.) Ach.

P. submontana Nadv. ex Hale

Parmeliella triptophylla (Ach.) Mül. Arg.

Parmelina quercina (Willd.) Hale

P. tiliacea (Hoffm.) Hale

Parmeliopsis hyperopta (Ach.) Arn.

Parmotrema chinense (Osbeck) Hale \& Ahti

Peccania coralloides Massal.

Peltigera aphtosa (L.) Willd.

$P$. canina (L.) Willd.

P. collina (Ach.) Schrad.

P. horizontalis (Huds.) Baumg.

P. leucophlebia (Nyl.) Gyeln.

P. malacea (Ach.) Funck.

$P$. polydactyla (Necker) Hoffm.

$P$. venosa (L.) Hoffm.

Peltula euploca (Ach.) Poelt

Pertusaria albescens (Huds.) Choisy \& Werner

$P$. alpina Hepp

P. amara (Ach.) Nyl.

$P$. aspergilla (Ach.) Laundon

$P$. caesioumbrina Eitner

$P$. chiodectonoides Bagl. ex Massal.

$P$. coccodes (Ach.) Nyl.

$P$. constricta Erichs.

P. coronata (Ach.) Th. Fr.

P. creatomma (Norm.) Zahlbr.

$P$. eitneriana Zahlbr.

$P$. geminipara (Th. Fr.) Knight. ex Brodo

$P$. glomerata (Ach.) Schaer.

$P$. hemisphaerica (Flk.) Erichs.

$P$. hymenea (Ach.) Schaer.

P. leioplaca DC.

P. ocellata (Wallr.) Körb.

P. oculata (Dicks.) Th. Fr.

$P$. pertusa (Weigel) Tuck.

$P$. rupestris (DC.) Schaer.

Phaeophyscia ciliata (Hoffm.) Moberg

Ph. endococcina (Körb.) Moberg

Phlyctis agelaea (Ach.) Flot.

Phylliscum demangeonii (Moug. \& Mont.) Nyl.

Physcia aipolia (Ehrh.) Fürnr.

Ph. stellaris (L.) Nyl.

Physconia distorta (With.) Laundon

Ph. perisidiosa (Erihsen) Moberg

Placocarpus schaereri (Fr.) O. Breuss

Placopsis gelida (L.) Linds.

Platismatia glauca (L.) W. Culb. \& C. Culb.

Pleospidium chlorophanum (Wahlenb.) Zopf.

$P$. flavum (Bellardi) Körb.

Pleurosticta acetabulum (Neck.) Ellis \& Lumbsch

Polyblastia abscondita (Nyl.) Arnold

P. cruenta (Körb.) James \& Swinscow

$P$. gelatinosa (Ach.) Th. Fr.

$P$. gothica Th. Fr.

P. melaspora (Taylor) Zahlbr.

P. sendtneri Krempelh.

$P$. sepulta Massal.

P. theleodes (Sommerf.) Th. Fr.

$\mathbf{R}$

E

E

V

V

E

Ex

E

V

$\mathbf{V}$

V

Ex

Ex

Ex

Ex

E

$\mathbf{E}$

ilesia

Poland 
Protoparmelia atriseda (Fr.) R. Sant. \& Wirth

Protothelenella leucothelia (Nyl.) Mayrh. \& Poelt

P. sphinctrinoides (Nyl.) Mayr. \& Poelt

Pseudephebe pubescens (L.) Choisy

Psora vallesiaca (Schaer.) Timdal [P. albilabra (Dufour) Körb. ssp. deceptoria (Nyl.) Clauz. \& Roux]

P. decipiens (Hedw.) Hoffm.

Psoroma hypnorum (Vahl) Gray

Psorotichia schaereri (Massal.) Arnold

Punctelia subrudecta (Nyl.) Krog

Pycnothelia papillaria (Ehrh.) Dufour

Pyrenocarpon flotowianum (Hepp) Trevisian

Pyrenula laevigata (Pers.) Arnold

$P$. nitida (Weigel) Ach.

P. nitidella (Flk.) Müll. Arg.

Pyrrhospora quernea (Dicks.) Körb.

Ramalina baltica Lettau

R. calicaris (L.) Fr.

$R$. capitata (Ach.) Nyl.

$R$. dilacerata (Hoffm.) Hoffm

R. farinacea (L.) Ach

$R$. fastigiata (Pers.) Ach.

$R$. fraxinea (L.) Ach.

R. pollinaria (Westr.) Ach.

R. polymorpha (Liljeblad) Ach.

$R$. thrausta (Ach.) Nyl.

Rhizocarpon disporum (Näg.) Müll. Arg.

$R$. eupetraeum ( $\mathrm{Nyl}$.) Arnold

$R$. grande (Flk. ex Flotow) Arnold

$R$. oederi (Weber) Körb.

$R$. petraeum (Wulf.) Massal.

$R$. simillinum (Anzi) Lettau

$R$. sorediosum Runem.

$R$. subgeminatum Eitner

$R$. saanaënse Räs. [R. sublucidum Räs.]

R. umbilicatum (Ramond) Flagey

$R$. viridiatrum (Wulf.) Körb.

Rimularia impavida (Th. Fr.) Hertel

Rinodina adspersa (Borr.) Laundon

R. archaea (Ach.) Arnold

$R$. atropallidula (Nyl.) Arnold

$R$. colobina (Ach.) Th. Fr

$R$. conradii Körb.

R. exigua (Ach.) Gray

$R$. fimbriata Körb.

R. milvina (Wahlenb. in Ach.) Th. Fr.

$R$. oxydata (Massal.) Massal.

$R$. polyspora $\mathrm{Th}$. Fr.

$R$. sophodes (Ach.) Massal.

R. teichophila (Nyl.) Arnold

R. tephraspis (Tuck.) Herre

$R$. trevisani (Hepp) Körb.

R. turfacea (Wahlenb.) Körb.

Sarcogyne clavus (DC.) Krempelh.

Sarcopyrenia gibba (Nyl.) Nyl.

Sarcosagium campestre (Fr.) Poetsch. \& Schied.

Schaereria cinereorufa (Schaer.) Th. Fr.

Schismatomma abietinum (Ehrh.) Massal.

Sclerophora nivea (Hoffm.) Tibel

Scoliciosporum perpusillum Körb.

Solorina crocea (L.) Ach.

S. saccata (L.) Ach.

Sphaerophorus fragilis (L.) Pers.

S. globosus (Huds.) Vainio

S. melanocarpus (Sw.) DC.

Sporastatia polyspora (Nyl.) Grum.

S. testudinea (Ach.) Massal.

Squamarina cartilaginea (With.) P. James

S. lentigera (Web.) Poelt

Staurothele ambrosiana (Massal.) Zsch.

S. fissa (Taylor) Zwackh

V

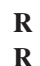

$\mathbf{R}$

$\mathbf{R}$

V

$\mathbf{R}$

$\mathbf{R}$

V

$\mathbf{R}$

I

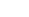

Ex

$\begin{array}{ll}\text { I } & \text { E } \\ \text { I } & \text { V }\end{array}$

I

$\mathbf{E}$

Ex

Ex

Ex

Ex

I

Ex

E

E

Ex 
S. fuscocuprea (Nyl.) Zsch.

S. guestphalica (Lahm ex Körb.) Arnold

S. hymenogonia (Nyl.) Th. Fr.

S. succedens (Rehm) Arnold

Steinia geophana (Nyl.) Stein

Stereocaulon condensatum Hoffm.

S. dactylophyllum Flk.

S. incrustatum Flk.

S. paschale (L.) Hoffm.

S. pileatum Ach.

S. tomentosum Fr.

Sticta fuliginosa (Hoffm.) Ach.

S. sylvatica (Huds.) Ach.

Strangospora ochrospora (Nyl.) Anderson

Strigula affinis (Massal.) R. C. Harris

S. stigmatella (Ach.) R. C. Harris

Synalissa symphorea (Ach.) Nyl.

Thamnolia vermicularis (Sw.) Schaer.

Thelenella modesta (Nyl.) Nyl.

Thelidium absconditum (Hrempelh.) Rabenh.

T. aenovinosum (Anzi) Arnold

T. amylaceum Massal.

T. aphanes Lahm

T. cataractarum (Hepp) Lönnr

T. decipiens Krempelh.

T. gisleri (Müll. Arg.) Zsch.

T. incavatum Mudd

T. saprophilum Servit

T. subabsconditum Eitner

Thelingya lignyota (Wahlenb.) Jorg. \& Henssen

Thelocarpon cinereum Eitner

T. impressellum $\mathrm{Nyl}$.

T. intermediellum $\mathrm{Nyl}$.

T. robustum Eitner

Thelomma ocellatum (Koerb.) Tibell

Thelotrema lepadinum (Ach.) Ach.

Thermutis velutina (Ach.) Flot.

Toninia aromatica (Sm.) Massal.

T. athallina (Hepp) Timdal

T. candida (Web.) Th. Fr.

T. sedifolia (Scop.) Timdal

Trapelia geochroa (Körb.) Hertel

T. mooreana (Carroll) P. James

Trapeliopsis gelatinosa (Flk.) Coppins \& P. James

T. viridescens (Schrad.) Coppins \& P. James

T. wallrothii (Flk. in Spreng.) Hertel \& G. Schneider

Umbilicaria crustulosa (Ach.) Fey

U. hyperborea (Ach.) Hoffm.

U. nylanderiana (Zahlbr.) H. Magn.

U. polyphylla (L.) Baumg.

$U$. proboscoidea (L.) Schrad.

$U$. propagulifera (Vainio) Llano

U. spodochroa (Hoffm.) DC.

U. vellea (L.) Hoffm.

Usnea ceratina Ach.

U. compacta Mot.

$U$. cornuta Körb.

$U$. diplotypus Vainio

$U$. filipendula Stirton

U. florida (L.) Web. ex Wigg.

$U$. glauca Mot.

U. hirta (L.) Web.

$U$. longissima Ach.

U. neglecta Mot.

$U$. rigida (Ach.) Röhl.

$U$. sorediifera Mot.

$U$. subfloridana Stirton

U. uncinulata Mot.

Varicellaria rhodocarpa (Körb.) Th. Fr.

Verrucaria acrotella Ach.

$\mathbf{R}$

$\mathbf{R}$

$\mathbf{R}$

$\mathbf{R}$

$\mathbf{R}$

$\mathbf{R}$

$\mathbf{R}$

V

Ex

E

V

V

V

R

R

R

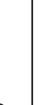

V

Ex

I

Ex

I

E

Ex

R

V

I

V

V

$\mathrm{v}$

$\mathrm{v}$

$\mathbf{v}$

V

$\mathbf{E}$

E

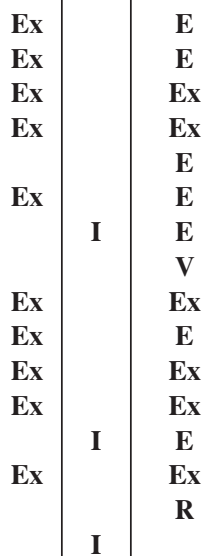


V. alutacea Zsch.

$V$. andesitica Servit

$V$. annulifera Eitner

$V$. apomeleana (Massal.) Hepp

$V$. aquatilis Mudd.

$V$. basaltica Servit

V. beltraminiana (Massal.) Trevisan

$V$. buellioides Servit

V. caerulea DC. in Lam \& DC.

$V$. cincta Hepp

V.foveolata (Flk.) Massal.

$V$. funckii (Spreng.) Zahlbr.

$V$. fusconigrescens $\mathrm{Nyl}$.

$V$. guestphalica Servit

V. hydrela Ach.

$V$. infumata Nyl.

V. krempelhuberi Lindau

$V$. latebrosa Körb.

$V$. longicollis (Eitner) Zahlbr.

$V$. macrostoma Dufour ex DC.

$V$. maculiformis Krempelh.

V. memmonia (Körb.) Arnold

V. mougeotii (Zsch.) Servit

$V$. murina Leighton

$V$. obnigrescens $\mathrm{Nyl}$.

$V$. praesudetica Zsch.

$V$. praetermissa (Trvis.) Anzi

V. procopii Servit

$V$. pulicaris Massal.

V.pulvinata Eitner

$V$. rheitrophila Zsch.

V. sublobulata Eitner ex Servit

V. submersella Servit

V. sylvatica Zsch.

$V$. tapetica Körb.

V. viridula (Schrad.) Ach

Vulpicida pinastri (Scop.) Ach.

Xanthoparmelia mougeotii (Schaer. ex Dietr.) Hale

Xanthoria calcicola Oxner

$X$. candelaria $($ L.) Th. Fr.

$X$. fallax (Hepp) Arnold

$X$. polycarpa (Hoffm.) Th. Fr. ex Rieber.

Xylographa minutula Körb.

X. paralella (Ach.) Behl. \& Desb.

$X$. vitiligo (Ach.) Laundon

Lower Silesia

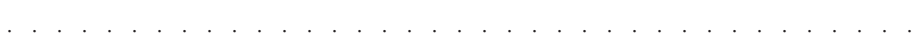

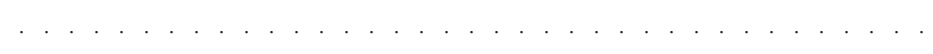

$\mathbf{R}$

$\mathbf{R}$

\begin{tabular}{c|c|c|c|c} 
& & I & \\
& Ex & & Ex \\
E & & I & V
\end{tabular}

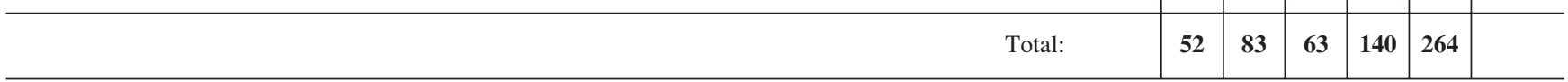

